\title{
Olivier GALLAND et Yannick LEMEL, Sociologie des inégalités
}

\section{Mathieu Lizotte}

\section{(2) OpenEdition \\ 1 Journals}

\section{Édition électronique}

URL : http://journals.openedition.org/ress/5485

DOI : $10.4000 /$ ress.5485

ISBN : $1663-4446$

ISSN : 1663-4446

Éditeur

Librairie Droz

Édition imprimée

Date de publication : 20 décembre 2019

Pagination : 294-296

ISSN : 0048-8046

Référence électronique

Mathieu Lizotte, "Olivier GALLAND et Yannick LEMEL, Sociologie des inégalités », Revue européenne des sciences sociales [En ligne], 57-2 | 2019, mis en ligne le 20 décembre 2019, consulté le 01 décembre 2020. URL : http://journals.openedition.org/ress/5485 ; DOI : https://doi.org/10.4000/ress.5485

Ce document a été généré automatiquement le 1 décembre 2020.

(c) Librairie Droz 


\title{
Olivier GALLAND et Yannick LEMEL, Sociologie des inégalités
}

\author{
Mathieu Lizotte
}

\section{RÉFÉRENCE}

Olivier GALLAND et Yannick LEMEL, 2018, Sociologie des inégalités, Paris, Armand Colin, $350 \mathrm{p}$.

1 Il n'est pas évident de délimiter un champ de recherche dans son entièreté en un seul ouvrage. Quiconque se prête à l'exercice ne pourra échapper aux critiques inéluctables concernant l'inclusion, l'exclusion ou l'omission de tels auteurs ou de telles perspectives. Olivier Galland et Yannick Lemel, qui ne sont pas à leur première collaboration, ont produit une remarquable synthèse de la sociologie des inégalités qui mériterait éventuellement une seconde édition. L'expérience prolongée dans un domaine donné conduit à jouir d'une expertise qui fait souvent l'objet d'un certain orgueil. Bien que l'on conçoive généralement ce type de manuel comme étant destiné aux étudiants, bon nombre de chercheurs bénéficieront de sa lecture, qu'ils soient récemment initiés au champ ou qu'ils s'y spécialisent. Pour ceux qui appartiennent à cette deuxième catégorie, cet ouvrage sera l'occasion de revisiter les ouvrages classiques, de découvrir de nouveaux auteurs et d'établir des liens insoupçonnés entre les deux. N'est-il pas utile à tout chercheur, de temps à autre, de sortir de la myopie de ses propres projets de recherche afin de former une vision globale de l'ensemble du champ? L'effort que les auteurs ont fourni afin de relater les débats actuels et passés sur les inégalités y concourent, en soulignant les éléments qui font l'objet de consensus ou de dissension, ou bien encore mènent à des impasses.

2 Les auteurs proposent de faire état de la sociologie des inégalités en six chapitres portant respectivement sur l'histoire des hiérarchies sociales, les théories classiques de la stratification et des inégalités, les définitions des inégalités, l'évolution des inégalités dans les sociétés industrielles, les inégalités entre groupe et les sentiments de justice, et 
la dynamique des inégalités. Le premier chapitre offre une synthèse ambitieuse sur l'histoire des hiérarchies sociales qui puise notamment dans la littérature anthropologique et historique. Le lecteur s'étonnera sans doute des développements sur les animaux sociaux (comme les hominidés) et la préhistoire humaine, mais ils participent en définitive d'une meilleure compréhension et contextualisation des inégalités contemporaines. Sans faire fi des exceptions notables, les études portant sur les inégalités ont mis davantage l'accent sur les causes conjoncturelles que les explications fondamentales (c'est-à-dire le pouvoir et la condition humaine). Ce déséquilibre s'explique en partie parce que ces dernières représentent un plus grand défi épistémologique. L'approche multidisciplinaire que les auteurs ont privilégiée dans ce chapitre, cependant, s'annonce plein de promesses afin d'éclaircir les questions de fond sur l'origine des hiérarchies sociales.

3 Le deuxième chapitre propose un survol des théories classiques de la stratification et des inégalités, dont le passage de la société d'ordre à la société de classes, les trois piliers, la reproduction, l'approche gradualiste, la mobilité sociale, etc. Il s'agit en quelque sorte du canon de la stratification avec lequel les experts dans le domaine seront familiers. La section qui relate le débat sur la mort des classes sera d'un intérêt notable à tout chercheur s'interrogeant sur la pertinence contemporaine et les limites de l'analyse de classe. La section consacrée au débat entre classe et genre permet de retracer la conscientisation de ce qui constitue sans doute la plus grande lacune dans l'histoire de la stratification. Bien que la condition féminine demeure un sujet négligé, le progrès observable ces dernières décennies est indéniable. Il suffit de consulter l'article de Joan Acker publié en 1973 dans The American Journal of Sociology («Women and Social Stratification: A Case of Intellectual Sexism », 78-4, p. 936-945), pour le constater.

4 La question importante, mais souvent esquivée, de la définition des inégalités fait l'objet du troisième chapitre. Non seulement les auteurs proposent une définition fort sensée, mais ils discutent et contrastent de nombreuses définitions philosophiques, économiques et politiques. Cette démarche permet de mieux cerner l'apport original d'une définition sociologique. Ils élaborent une définition des inégalités d'inspiration wébérienne tout à fait convenable tenant sur trois éléments : une inégalité est 1) une différence dans l'accès à des éléments ayant de la "valeur " (au sens large, c'est-à-dire à la fois matériel et symbolique); 2) une différence entre des personnes ou des groupes ; 3) une différence jugée, au moins selon certains, comme injustifiée et pouvant faire l'objet de réforme. Leur définition tient compte intrinsèquement du fait que toute inégalité est liée aux représentations sociales ainsi qu'aux conflits de groupes ne partageant pas les mêmes perspectives.

5 Le quatrième chapitre offre un portrait empirique très détaillé de l'évolution des inégalités économiques au cours $\mathrm{du} \mathrm{xx}^{\mathrm{e}}$ siècle tout en soulignant les facteurs explicatifs (transformation de l'économie, changement de rémunération, rendement respectif du capital et du travail, changement démographique, femme au marché du travail, etc.). Les auteurs admettent eux-mêmes qu'il n'est pas suffisant de se focaliser uniquement sur le revenu. Cela se justifie cependant à la lumière de l'importance à la fois théorique, historique et contemporaine des inégalités économiques. Le cours des inégalités économiques constitue une trame de fond permettant de mieux contextualiser les autres formes d'inégalités qui sont abordée dans l'ouvrage, comme les conditions de travail, la mobilité sociale, l'éducation et la santé. Le choix des pays y est limité, mais il 
sert à faire un portrait général des inégalités au sein des pays développés. Un effort a été fait pour inclure plusieurs figures qui remontent au début $d u x^{e}$ siècle. Les auteurs ont aussi pris soin de rappeler que le bilan des inégalités ne coïncide pas forcément entre les mesures absolues ou relatives. Ce dernier point mériterait d'ailleurs d'être davantage discuté dans l'ouvrage, car il n'est pas suffisamment souligné dans la littérature scientifique, ni bien compris dans le débat public. Quelle que soit l'évolution des inégalités en terme relatif, le revenu national per capita a augmenté considérablement en valeur absolue dans la majorité des pays de l'OCDE au cours du dernier siècle et cela n'est pas sans conséquence sur le plan politique. Ce constat luimême offre un argument intéressant, même s'il n'est pas original, afin d'expliquer l'essor des revendications identitaires aux dépens des revendications de classe.

6 Est abordée, par la suite, dans le cinquième chapitre, la question des inégalités entre groupes sociaux et les sentiments de justice. Les auteurs se concentrent ici sur les principales formes d'inégalités qui ne relèvent pas de la classe sociale et, notamment, celles liées aux catégories socioprofessionnelles, au chômage, au genre, à l'orientation sexuelle, au cycle de vie, à la dimension spatiale, à l'immigration, etc., ainsi qu'à l'intersectionnalité. Ce faisant, ils cernent bien les nouvelles orientations qui ont émergé dans le champ depuis le milieu des années 1990 et qui ont imposé un virage privilégiant une sociologie des inégalités sociales en lieu et place d'une analyse strictement en termes de stratification. La section sur les sentiments de justice pourrait faire l'objet d'un chapitre en soi étant donné l'importance de la dimension subjective et des tensions idéologiques entre les groupes dans leur définition des inégalités.

7 Enfin, le sixième et dernier chapitre porte sur la dynamique des inégalités. Les auteurs y survolent les principaux thèmes concernant la stase ou le changement des inégalités, à savoir : les mouvements sociaux, la reproduction - à travers le rôle de l'école et de la famille -, les trajectoires individuelles et le rôle de l'État face aux inégalités. Il ne suffit pas cependant de documenter les inégalités, ni de les exposer à la lumière, mais encore de les expliquer. La plupart de ces mécanismes de reproduction sont bien connus et ont fait l'objet de plusieurs études. De loin le mécanisme le moins étudié est le lien intrinsèque entre les inégalités et la normativité. Les auteurs font bien de reconnaître à la fois que toute différence d'accès aux biens sociaux n'est pas forcément une inégalité et que la distinction différence/inégalité est contingente à de nombreux facteurs, dont l'idéologie n'est pas le moindre, qui ont été trop peu étudiés.

8 Les auteurs concluent l'ouvrage avec un plaidoyer en faveur d'un cadre théorique unificateur pour lier les perspectives de la classe et de l'identité. Dans la pratique, bon nombre de sociologues ont subsumé la question de la reconnaissance et du mépris sous la notion de statut, mais l'intégration théorique mérite encore des travaux approfondis. Jusqu'à présent, il n'y a pas eu un seul ouvrage sociologique de taille qui tente d'intégrer ces deux notions. Espérons que cet appel sera entendu et que certains relèveront le défi. D'ici là, le temps est venu de cesser d'opposer la classe à l'identité comme si reconnaître l'un revenait à désavouer l'autre. Comme le soutiennent Serge Paugam et Marie Plessz dans ce même numéro (RESS, 57-2), la sociologie des classes demeure bien vivante à l'intérieur de la sociologie des inégalités. Les autres formes d'inégalités n'ont pas éclipsé la classe, mais lui offrent, au contraire, un regard complémentaire et nécessaire. 


\section{AUTEURS}

MATHIEU LIZOTTE

University of Ottawa 